\title{
Pulmonary Sarcoidosis following Etanercept Treatment for Ankylosing Spondylitis: A Case Report and Review of the Literature
}

\author{
A. Majjad (D), ${ }^{1}$ A. Bezza, ${ }^{1}$ A. Biyi, ${ }^{2}$ M. R. El Ochi, ${ }^{3}$ and A. El Maghraoui ${ }^{1}$ \\ ${ }^{1}$ Rheumatology Department, Mohammed V Military Academic Hospital, Faculty of Medicine and Pharmacy, \\ Mohammed V University, Rabat, Morocco \\ ${ }^{2}$ Nuclear Medicine Department, Mohammed V Military Academic Hospital, Faculty of Medicine and Pharmacy, \\ Mohammed V University, Rabat, Morocco \\ ${ }^{3}$ Anatomic Pathology Department, Mohammed V Military Academic Hospital, Faculty of Medicine and Pharmacy, \\ Mohammed V University, Rabat, Morocco
}

Correspondence should be addressed to A. Majjad; drmajjad@gmail.com

Received 30 September 2017; Revised 29 November 2017; Accepted 20 December 2017; Published 23 January 2018

Academic Editor: Tsai-Ching Hsu

Copyright (c) 2018 A. Majjad et al. This is an open access article distributed under the Creative Commons Attribution License, which permits unrestricted use, distribution, and reproduction in any medium, provided the original work is properly cited.

Antitumor necrosis factor therapies have revolutionized the treatment of some inflammatory diseases. However, the use of these agents is associated with the development of many paradoxical autoimmune diseases. Less well-recognized is the association with sarcoidosis. We report a 55-year-old female with long-standing ankylosing spondylitis who developed persistent dry cough and dyspnea while receiving etanercept therapy. High-resolution computed tomography scanning showed mediastinal lymphadenopathy and multiple nodules in both lung fields developed two months after the administration of etanercept. Lymph node biopsy was not practicable. Histopathological examination of minor salivary gland biopsy revealed noncaseating granulomata, and the serum angiotensin-converting enzyme was very elevated. All infectious studies were negative. Etanercept was discontinued plus a course of corticosteroids with a clinical improvement, and a follow-up high-resolution computed tomography scanning 4 months later showed evident regression of mediastinal lymph nodes and pulmonary nodules. Potential pathogenic mechanisms of this paradoxical effect of tumor necrosis factor-alpha blocking agents are discussed.

\section{Background}

Sarcoidosis is a rare granulomatous disease mainly affecting the lungs and lymph nodes. Its etiology remains unknown [1]. However, there are a small number of literatures possible that involve tumor necrosis factor-alpha blocking agents (anti-TNF- $\alpha$ ), especially etanercept, in the development of pulmonary sarcoidosis [2]. Our case report supports possible relationship between etanercept therapy and the development of sarcoid-like granulomatosis.

\section{Case Report}

A 66-year-old female patient without medical history or professional exposures was diagnosed with ankylosing spondylitis 12 years ago. She had formerly responded to nonsteroidal inflammatory drugs. In 2006, she started treatment with infliximab, due to poor therapeutic response to various NSAIDs. After eight years of remission, the patient had repeated flares of ankylosing spondylitis symptoms with increased inflammatory back pain. In August 2015, due to persistent clinical activity with increased acute-phase reactant levels (C-reactive protein level of $16 \mathrm{mg} / \mathrm{l})$, the treatment with etanercept at $25 \mathrm{mg}$ twice weekly was started. Following national guidelines to exclude latent tuberculosis, mycobacterium tuberculosis research in sputum and interferon-gamma test were negative and a chest radiograph was normal. Two months after etanercept therapy onset, the patient developed persistent dry cough and dyspnea without fever or night 

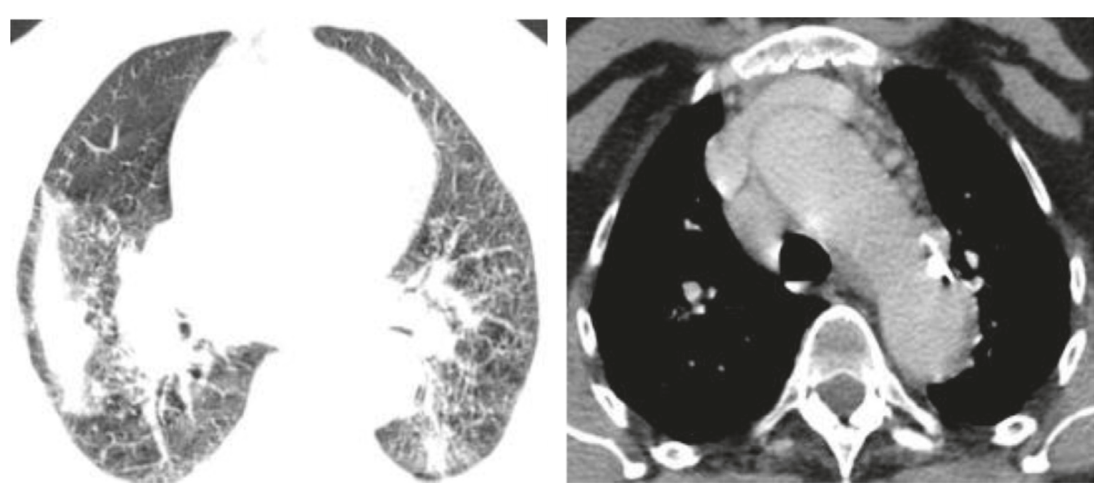

Figure 1

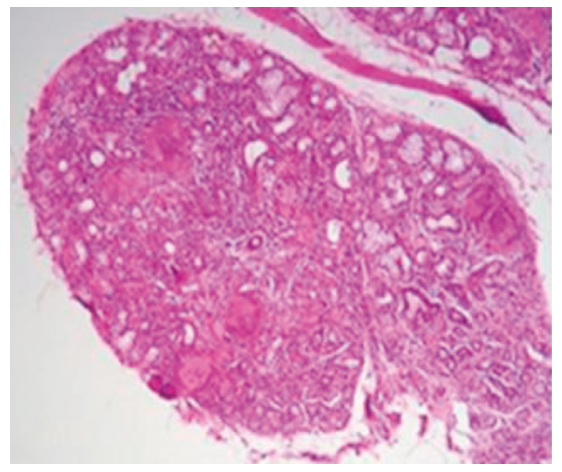

FIGURE 2
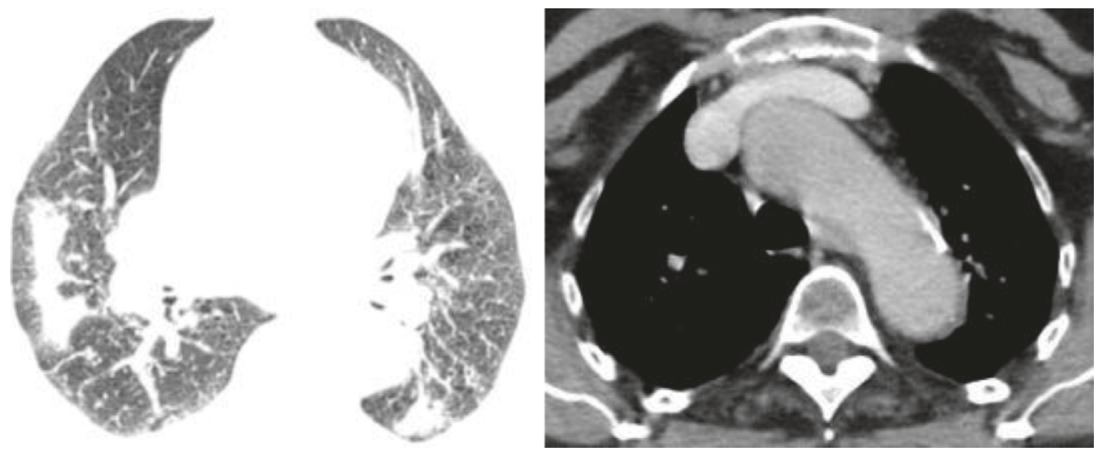

Figure 3

sweating. Physical examination was unremarkable apart from tachypnea and severe spinal stiffness. Highresolution computed tomography (HRCT) scanning showed mediastinal lymphadenopathy and multiple nodules in both lung fields (Figure 1). Echocardiography was normal. The blood analysis demonstrated ESR $35 \mathrm{~mm} / \mathrm{h}$, CRP $14 \mathrm{mg} / \mathrm{l}$, and normal renal and hepatic function tests. Sputum culture and polymerase chain reaction and interferon-gamma release assays were negative. Viral serology (human immunodeficiency virus, hepatitis $B$ virus, and hepatitis $C$ virus) and syphilis serology were negative. Serum angiotensin-converting enzyme was very elevated at $147 \mathrm{UI} / \mathrm{ml}$ (reference range 8-52 UI/ml). Calcemia and calciuria tests were normal.
The pulmonary function test showed restrictive ventilatory alteration. It was not possible to perform neither bronchoscopy nor thoracoscopy because of respiratory failure and severe ankylosis of the cervical spine. Histopathological examination of minor salivary gland biopsy revealed a chronic granulomatous sialadenitis without caseous necrosis (Figure 2). Diseases showing similar histologic changes were excluded, and a diagnosis of sarcoidosis was made. Etanercept was discontinued plus a course of corticosteroids with $40 \mathrm{mg}$ /day of prednisone for two months. An evident improvement of cough and dyspnea was seen. Follow-up HRCT 4 months later showed regression of mediastinal lymph nodes and pulmonary nodules (Figure 3). 


\section{Discussion}

Sarcoidosis is a multisystem disorder characterized by the accumulation of lymphocytes, mononuclear phagocytes, and noncaseating granulomas in involved tissues. Etiology of sarcoidosis is still not identified. The role of viral, bacterial, and parasitic infections is not clearly determined. The role of some drugs and environmental exposure are still considered [3]. Due to unknown causes, active macrophages and lymphocytes are accumulated in this or that organ and produce increased number of interleukins, TNF- $\alpha$. The TNF- $\alpha$ is the key cytokine that participates in the formation of granulomas in sarcoidosis $[3,4]$.

At present, there are five anti-TNF- $\alpha$ agents currently available: four monoclonal antibodies (infliximab, adalimumab, golimumab, and certolizumab) and a soluble TNF$\alpha$ receptor fusion protein (etanercept) [5]. Anti-TNF- $\alpha$ agents, especially infliximab and sometimes adalimumab, are effective in refractory sarcoidosis $[6,7]$. However, there have been a few cases of sarcoidosis occurring during antiTNF- $\alpha$ therapy. In a recent review of literature published on January 2016, SimJae Kyeom et al. found 59 cases of sarcoidosis occurring during anti-TNF- $\alpha$ therapy in the medical literature. Thirty-seven $(61.6 \%)$ occurred after etanercept, 13 (21.6\%) after adalimumab, and 9 (15\%) after infliximab. In this study, twenty-eight had rheumatoid arthritis. The lung and/or lymph node were the most commonly affected organs (64\%), followed by the skin (37\%) and the eye (15\%). Prognosis seems to be favorable since 52 of 59 cases displayed a partial or complete resolution after antiTNF- $\alpha$ withdrawal with or without steroids [2].

The pathogenesis of sarcoidosis induced by anti-TNF- $\alpha$ remains unclear. However, some potential hypotheses have been proposed. Mainly followed hypothesis is that sarcoidosis is of an infectious origin favored by an immunosuppressant effect of anti-TNF- $\alpha$, especially with some microorganisms that are implicated in the development of sarcoidosis (Propionibacterium acnes and Propionibacterium granulosum) [8]. Another hypothesis is that the disease is an immunological disorder, with an imbalance in Th17 lymphocytes and Treg lymphocytes, favoring Th17 and formation of granulomas via the increased expression of IL17 and TNF- $\alpha$. Currently, there are many reports supporting this latter hypothesis [9-11]. Indeed, more than $60 \%$ of the reported cases occurred with etanercept. There are two hypotheses that may explain this greater association: on one hand, the difference in pharmacological proprieties between the synthesized anti-TNF- $\alpha$ receptor and monoclonal antibodies: the antibodies neutralize both soluble and membrane forms of TNF- $\alpha$, whereas etanercept neutralizes just the soluble form. This partial inhibition permits the redistribution of TNF- $\alpha$ in areas of elevated concentration such as the lungs and lymph nodes [11]. On the other hand, several studies suggest that monoclonal antibodies, in contrast to etanercept, inhibit the expression of IL17 and INF-gamma, cytokines strongly involved in granuloma formation [12]. Taking into account that it is not the only case of sarcoidosis developed with the anti-TNF therapy, the pathogenesis of granulomas under the effect of the therapy needs to be studied. Besides, it is possible that similar complications of anti-TNF therapy may be developed in carriers of HLA-A1, B8, and B13 antigens as it is known that those antigens are met more frequently in sarcoidosis patients compared to the general population [13]. In summary, it is important for clinicians to be aware of this rare complication of anti-TNF therapy. Sarcoidosis granulomas may be formed not only in the intrathoracic lymphatic nodes, lungs, and salivary glands but in other organs too (liver, spleen, eyes, heart, skin, etc.). If the biopsy of the affected organ is difficult, minor salivary gland biopsy may be an excellent alternative and help in diagnosis [11, 13].

\section{Conclusion}

Pulmonary sarcoidosis is an uncommon effect of anti-TNF therapy. The pathogenic mechanism is unclear. Minor salivary gland biopsy may help in diagnosis. The management requires discontinuing anti-TNF plus a course of steroids.

\section{Consent}

Written informed consent was obtained from the patient for publication of this case report.

\section{Conflicts of Interest}

No conflicts of interest were declared by the authors.

\section{Acknowledgments}

The authors thank the patient and her family.

\section{References}

[1] N. Unterstell, A. L. Bressan, L. A. Serpa, and A. C. Gripp, "Systemic sarcoidosis induced by etanercept: first Brazilian case report," Anais Brasileiros de Dermatologia, vol. 88, no. 6, pp. 197-199, 2013.

[2] J. K. Sim, S. Y. Lee, J. J. Shim, and K. H. Kang, "Pulmonary sarcoidosis induced by adalimumab: a case report and literature review," Yonsei Medical Journal, vol. 57, no. 1, pp. 272-273, 2016.

[3] J. Müller-Quernheim, A. Prasse, and G. Zissel, "Pathogenesis of sarcoidosis," La Presse Médicale, vol. 41, no. 6, pp. e275e287, 2012.

[4] N. W. Lukacs, S. W. Chensue, R. M. Strieter, K. Warmington, and S. L. Kunkel, "Inflammatory granuloma formation is mediated by TNF-alpha-inducible intercellular adhesion molecule-1," Journal of Immunology, vol. 152, no. 12, pp. 5883-5889, 1994.

[5] A. Mounach and A. El Maghraoui, "Efficacy and safety of adalimumab in ankylosing spondylitis," Open Access Rheumatology, vol. 6, pp. 83-90, 2014.

[6] R. P. Baughman, M. Drent, M. Kavuru et al., "Infliximab therapy in patients with chronic sarcoidosis and pulmonary involvement," American Journal of Respiratory and Critical Care Medicine, vol. 174, no. 7, pp. 795-802, 2006.

[7] N. J. Sweiss, I. Noth, M. Mirsaeidi et al., "Efficacy results of a 52-week trial of adalimumab in the treatment of refractory sarcoidosis," Sarcoidosis, Vasculitis, and Diffuse Lung Diseases, vol. 31, no. 1, pp. 46-54, 2014. 
[8] Y. Eishi, M. Suga, I. Ishige et al., "Quantitative analysis of mycobacterial and propionibacterial DNA in lymph nodes of Japanese and European patients with sarcoidosis," Journal of Clinical Microbiology, vol. 40, no. 1, pp. 198-204, 2002.

[9] D. E. Furst, R. Wallis, M. Broder, and D. O. Beenhouwer, "Tumor necrosis factor antagonists: different kinetics and/or mechanisms of action may explain differences in the risk for developing granulomatous infection," Seminars in Arthritis and Rheumatism, vol. 36, no. 3, pp. 159-167, 2006.

[10] C. Prior and P. L. Haslam, "Increased levels of serum interferon-gamma in pulmonary sarcoidosis and relationship with response to corticosteroid therapy," American Review of Respiratory Disease, vol. 143, no. 1, pp. 53-60, 1991.

[11] C. Vigne, F. Coury, Y. Pacheco, and J. Tebib, "AB0452 sarcoidosis: an underestimated and potentially dramatic side effect of anti-TNF-a," Annals of the Rheumatic Diseases, vol. 71, no. 3, p. 663, 2013.

[12] É. Toussirot, "Sarcoïdose induite par les anti-TNFa," Commentaires à propos l'article de Vigne $\mathrm{C}$ et al. (Joint Bone Spine 2013; 80: 104-107)," Revue du Rhumatisme, vol. 80, no. 6, pp. 631-632, 2013.

[13] K. Bellil, I. Chelly, G. I. Ben et al., "Salivary gland biopsy: experience of La Rabta Hospital's pathology department," La Tunisie Medicale, vol. 85, no. 1, pp. 64-66, 2007. 


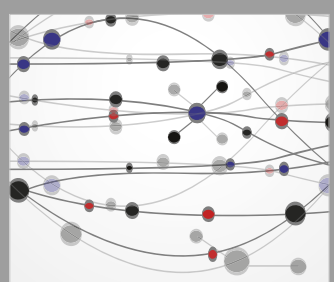

The Scientific World Journal
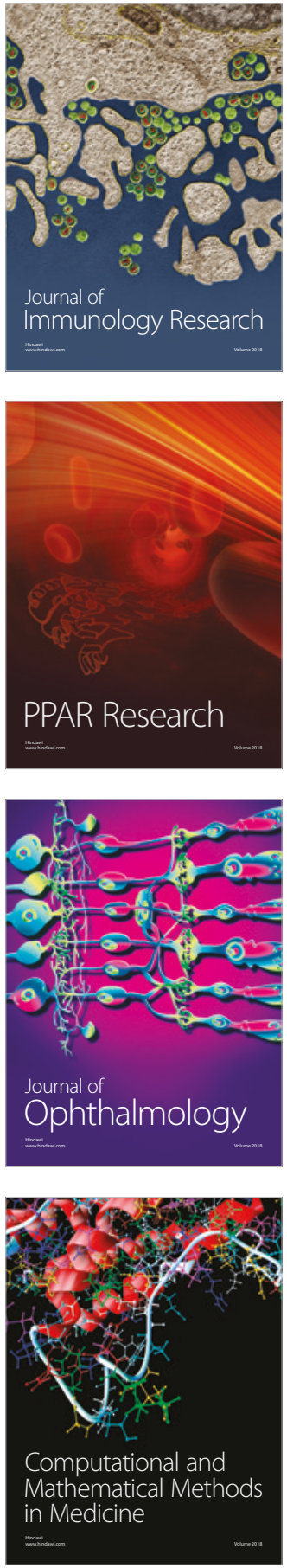

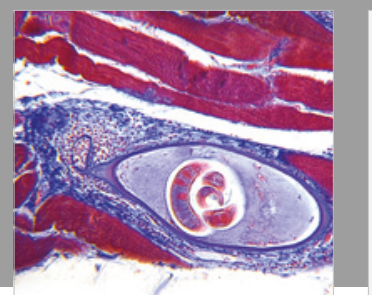

Gastroenterology Research and Practice

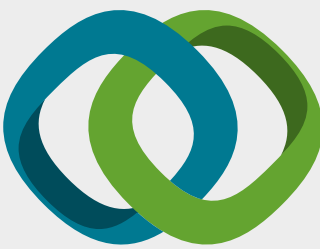

\section{Hindawi}

Submit your manuscripts at

www.hindawi.com
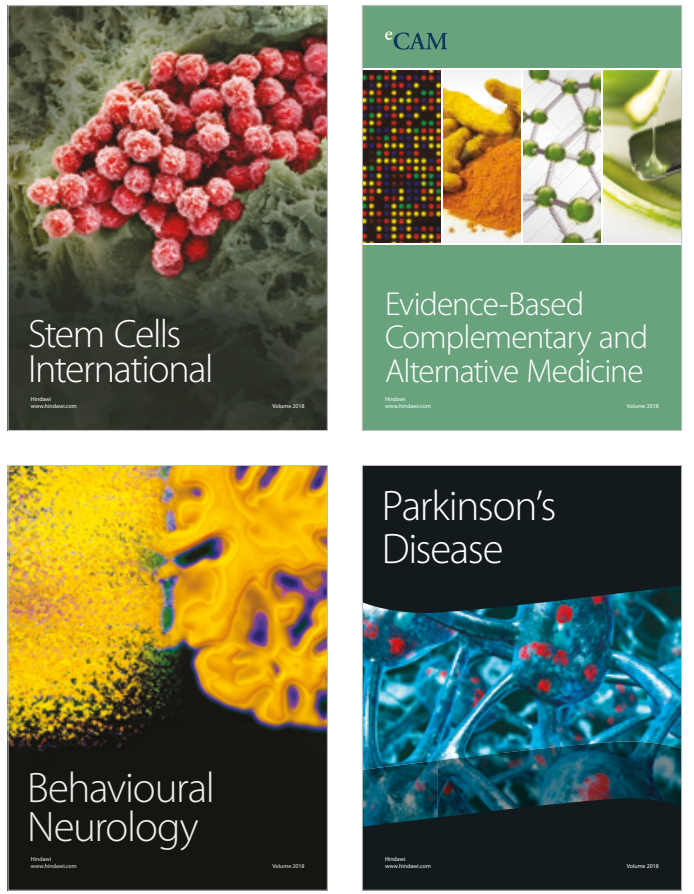

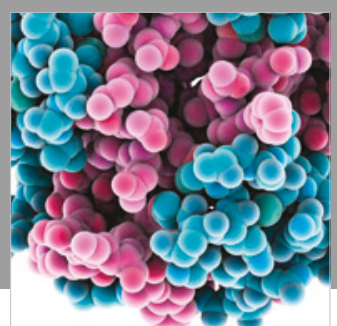

ournal of

Diabetes Research

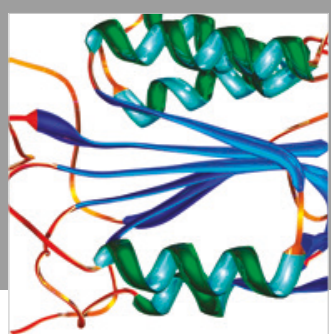

Disease Markers
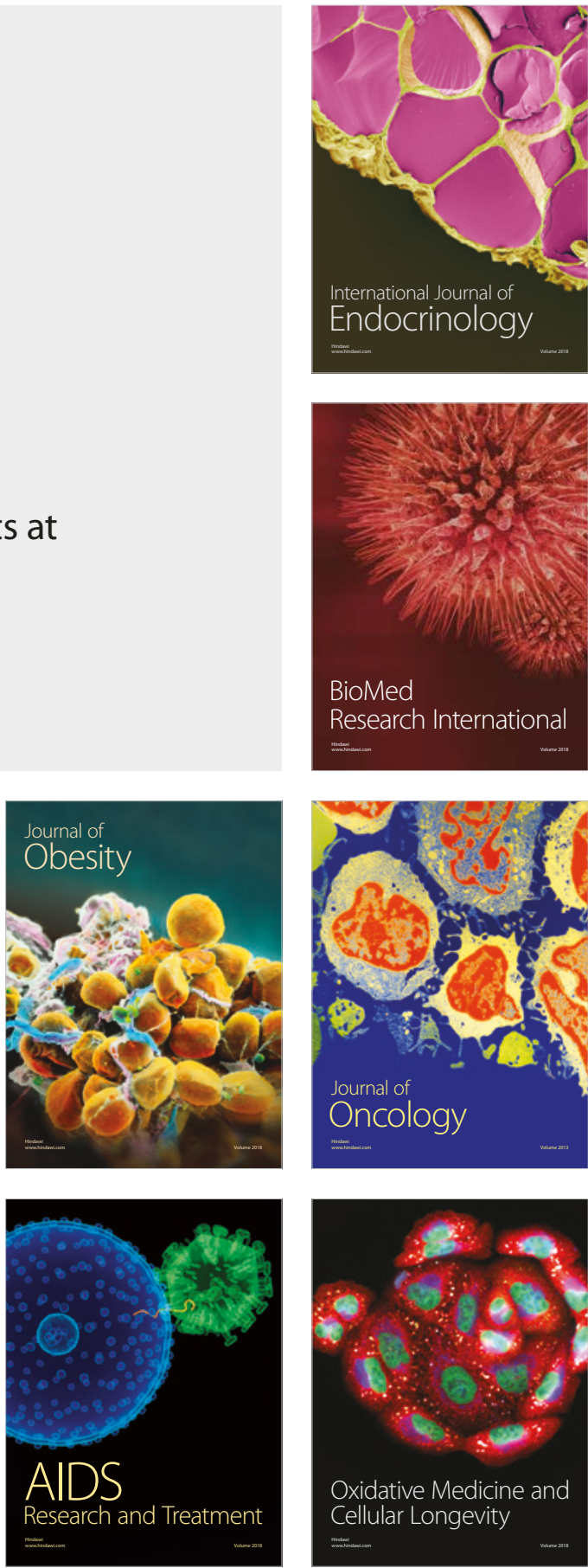